\title{
BARRIERS TO EFFECTIVE KNOWLEDGE MANAGEMENT: ACTION RESEARCH MEETS GROUNDED THEORY [CASE STUDY]
}

\author{
David G. Wastell \\ Information Systems Institute, Salford University, Manchester, M5 4WT, UK \\ Tel.: + 44161295 5102, Fax: + 441617458169 \\ D.Wastell@salford.ac.uk
}

\begin{abstract}
Despite evidence that the majority of knowledge management (KM) initiatives miscarry, there has been a paucity of critical, in-depth research into the causes of failure. In this paper, an action research (AR) project is described in a major motor manufacturer (WWM) focusing on one of their key knowledge processes (Design for Manufacturabilty, DFM). Intensive field work using a grounded theory methodology led to the production of a model relating the effectiveness of knowledge generation and use to a set of success factors. Many of the factors resonate with key issues described in previous KM research; for example, the need for a shared knowledge repository, a formal KM process, and a culture disposed towards knowledge sharing and re-use. The model has provided a rigorous platform on which to design interventions to improve the DFM process in WWM. Whilst technical changes (creating infrastructure, defining processes) are readily feasible, bringing about cultural change is less tractable and process improvements in WWM have tended to focus on the former rather than the latter. As well as providing insights into KM success and failure, the paper demonstrates the valuable role that grounded theory can make to the practice of action research in IS. In relation to the problem-solving aspect of AR, grounded theory can help to inform the design of organisational interventions and the evaluation of their impact; in relation to research, GT adds rigour and method to the theory building process, thereby raising the status of AR as a research tool.
\end{abstract}

\section{INTRODUCTION}

Knowledge management $(\mathrm{KM})$ is the subject of intense topical concern in both academic and practitioner communities. Knowledge, skills and creativity are increasingly seen by business as the key to competitive success. Although many organisations have launched initiatives to develop and exploit their knowledge resources (Davenport, 1998; Swan et al, 1999), many of these projects have been unsuccessful (Schultze and Boland, 2000) with failure rates of over $80 \%$ being quoted by some authors (Storey and Barnett, 2000). On the academic side, KM has attracted much recent interest; the research literature has burgeoned, with the issue of failure naturally emerging high on the agenda. Some of this research has taken the form of high-level surveys focusing on success factors and aspects of best practice. These surveys have typically involved the elicitation of general reflections from senior KM practitioners through non-intensive methods, such as telephone interviews (e.g. Davenport, 1998) or questionnaire-based approaches, such as the Delphi study of Holsapple and Joshi (2000). Case studies focusing on success/failure have been also been reported (e.g. 
Shani et al., 2000; Gupta and Govindajaran, 2000; Damodaran and Olphert, 2000; Storey and Barnett, 2000). However, these studies often lack critical depth and/or methodological rigour. Typically, results appear as lists of factors, with no clear link to underlying data in terms of cited evidence or an explicit method of theory generation. An exception is the ethnographic study of Schultze and Boland (2000). Though impressive in terms of empirical rigour, this otherwise exemplary study is, however, limited in its theoretical novelty.

The purpose of this paper is to describe an in-depth action research (AR) project focused on the vicissitudes of a key knowledge management process in a major manufacturing company, and on the development of a set of measures to remedy some of these problems. The work is distinctive in two key ways in relation to previous empirical research on KM: first, it is an action research project (i.e. the research was motivated at a practical level towards the design and evaluation of an ameliorative intervention); second, it makes use of a grounded theory (GT) methodology (Glaser and Strauss, 1967) to provide a rigorous basis for the development of theory. The paper will describe and discuss the underlying technical, cultural and organisational obstacles that limited the degree to which the knowledge process attained its desired objectives in WWM, presenting the results in the form of a grounded theory of KM effectiveness. This theory formed the basis for a set of recommendations for improving the KM process, some of which have now been implemented.

The paper aspires to make two contributions. As well as contributing theoretically to the literature on KM (by providing insights into obstacles and remedies), the author also seeks to make a general methodological point regarding the use of action research (AR) in IS. Whilst AR has its votaries (Baskerville and WoodHarper, 1998), the disparaging view persists that it is "little more than consultancy" (McKay and Marshall, 2001). McKay and Marshall attribute this to an overemphasis on the problem-solving aspects of AR (the practitioner interest) at the expense of its role as a vehicle for theory development and evaluation (the research interest). By providing a rigorous method for deriving theory, GT has potential to strengthen the research role of AR and hence to elevate its epistemological status in IS. By providing a rigorous platform on which to design and evaluate interventions, the problem-solving role of AR is also strengthened.

\section{KNOWLEDGE MANAGEMENT IN NEW PRODUCT DEVELOPMENT}

\subsection{Background}

The industrial partner in this project was in the automobile sector and will be known as WWM (World Wide Motors). WWM is one of the world's largest automotive manufacturing corporations, employing more than 300,000 people worldwide with a global presence in more than 200 countries. This project was concerned with the management of knowledge in WWM's design process for new vehicles, specifically with the efficacy of a key KM tool, namely Design for Manufacturability (DFM). Effective knowledge management in the product design process is now recognised as the key to success throughout the manufacturing sector (Ramesh and Timawa, 1999; Lynn et al, 2000), and there are acclaimed examples of its use in the automobile industry (McDermott and O'Dell, 2001). DFM plays a key part in WWM's engineering design strategy and has been the subject of heavy investment over several years, supported by vigorous internal promotion from the highest levels in the company. Nonetheless serious doubts were being voiced as to its efficacy. These concerns focused on the quantity and quality of design ideas generated and the degree to which they were carried through into the engineering design process, and ultimately implemented in the manufactured vehicle.

\subsection{Design for Manufacturability}

However innovative a design might be, it is vital that it be manufacturable if the enterprise is to survive and prosper. This truism is the driving force behind DFM. With any artifact (be it software or a new vehicle) the cost of correcting design flaws increases exponentially as the product life-cycle unfolds: changing a design at 
the conceptual stage can cost little more than damage to the ego, correcting an error once a million items have been sold can compromise the entire enterprise. The aim of DFM is to facilitate the sharing of design knowledge amongst the various engineering specialists who participate in vehicle design, such that products are designed not only to satisfy technical requirements but also to optimise the product's buildability, i.e. to minimise manufacturing costs and lead times, to address ergonomic concerns etc.

\subsection{The DFM Process in WWM}

DFM is an integral part of WWM's vehicle development process; it is seen as a knowledge tool enabling a broad range of factors that bear on the quality of a design to be addressed as early as possible within the design process. At the heart of the DFM process is the DFM workshop; this is the main forum in which design knowledge is shared and new design concepts elaborated. It is the fulcrum of the entire process and constitutes the primary focus of the present work.

At the outset of all vehicle programmes, a series of DFM workshops is planned. DFM will be applied to any sub-system (e.g. exterior lights, head-liners) which involves significant new engineering content or where there are concerns arising from the historical performance of that component. The latter could reflect any aspect ranging from assembly problems to difficulties in servicing the vehicle once it has been sold. The workshops are seen as an important opportunity to influence product design to take into account multiple engineering objectives: to reduce assembly time, to reduce the number of parts, to reduce mass, to optimise manufacturing investment, to improve reliability and serviceability. Of the order of 100 workshops are held for a typical vehicle programme.

Two individuals play key roles in DFM: the DFM engineer (a DFM specialist who will facilitate the workshop and generally support the DFM process from beginning to end) and the Design Release Engineer (DRE), i.e. the individual in overall charge of the design work for that component/sub-system from inception to fruition. Some weeks in advance of the planned date for a workshop, the DFM engineer and the DRE meet to discuss the detailed content of the workshop and to decide who should be invited. Preparatory work is then set in train so that any required analytical work is performed in advance (e.g. analyses of assembly sequences) and to ensure that any materials that are required at the work-shop are ready and available (e.g. mock-ups).

The workshop itself conforms to a standard format, though the duration can vary from half a day to a week. The first phase is essentially one of information sharing: the DFM engineer opens the workshop, followed by the DRE who introduces the topic in terms of the sub-system itself, current design ideas, workshop aims etc. General vehicle and manufacturing requirements are then reviewed by appropriate specialists, and opportunities for design improvement are addressed from a range of quality perspectives (robust engineering, dimensional engineering, feedback from assembly plants). The second phase involves brain-storming around ideas for improving the baseline design, and the workshop concludes with an evaluation of different design solutions involving the combination of brainstorming options. An action list to address unresolved issues is drawn up. Following the workshop, a report is circulated summarising its proceedings and outcome.

\subsection{Organisational Support for DFM}

The DFM community in WWM is a large one. At the time of the study, it consisted of a substantial central group (over 20 individuals) who provided, inter alia, DFM training and consultancy to the divisions, and operated a central benchmarking facility ("tear-downs"of rival vehicles). Responsibility for implementing DFM was devolved to the different divisions of the Company, each of which had a DFM support team. There were several such divisions: small car, large car, truck etc. In the latter division, for instance, the support team consisted of around 8 staff. DFM engineers in these teams were responsible for drawing up the DFM schedules for their divisions and for supporting the workshop process. A DFM user group was also in existence which operated as a forum for sharing ideas and best practices, and for considering DFM 
improvements through new tools and process innovations. There was also a DFM Policy Group and a Steering Committee.

\subsection{RESEARCH METHODOLOGY}

\subsection{Theoretical Sampling and the Grounded Theory Approach}

As with any new idea, the original purity and coherence of the GT concept (Glaser and Strauss, 1967) has become disfigured by schismatic dispute as to its true character and approved practice (Goulding,1998). Nonetheless, certain core ideas remain clearly discernible, principally the intimate dialectical interplay between data collection and theory production that forms the heart of the GT process. The reader is referred to the two classic texts in the area (Glaser and Strauss, 1967; Strauss and Corbin, 1998) for a detailed introduction; here we have space only for a few words to convey something of the spirit of the approach.

Grounded theory is simply theory that arises directly from field data and which, in turn, reflexively guides the research process. Once a research topic has been delineated, the process of data gathering is set in train. Characteristically, the researcher eschews taking an a priori theoretical position, instead leaving the data to "speak for itself" in generating theory. Theory is built by continuously inspecting data for new concepts (the process of open-coding, i.e. attaching labels to data). General categories are derived from this coding process and new data is "constantly compared" against these categories (are the existing ones adequate, are new ideas required?). Categories are elaborated, generalised and internally consolidated (axial coding) as the research process unfolds and an overall theoretical structure progressively takes shape. Adaptability and pragmatism are the hallmarks of GT. Data, for instance, is typically qualitative but, laudably, quantitative methods are not religiously debarred (Strauss and Corbin, 1998). The emergent nature of theory means that data gathering (sampling) must respond reactively as new theoretical themes emerge. All cannot be planned in advance; the researcher must follow her "investigative nose", in a process known as theoretical sampling. GT is not embarrassed by the fuzzy, shifting nature of the research process, indeed it embraces and celebrates it.

\subsection{Field Work: Practical Aspects}

The field-work for this project was carried out in the summer of 1999. The practical aims were fourfold: 1) to gain a working familiarity with DFM as it operated in WWM, within the broader context of the vehicle design process; 2) to make an assessment of the current efficacy of the DFM process; 3 ) to identify set of key success/risk factors that appeared to bear on this and to elaborate these as grounded theory; 4) to produce a set of recommendations for improving the process.

Two episodes of field-work were carried out. The first involved non-participant observation of a sample of DFM workshops. Six were attended: 3 in the Truck Division and 3 dealing with medium sized cars. The workshops followed either a half-day or a one-day format. Opportunistic interviews with DFM facilitators and workshop participants were held and key staff in the central support group were also interviewed. Initial ideas were codified and presented in an interim report to the DFM user group for validation and general feedback.

The second episode involved a series of intensive interviews with key DFM stakeholders, drawn from participants at the workshops the author attended. Selection of interviewees and topics were initially guided by the themes in the interim report, but the researcher also responded to any new ideas that emerged. The general goals of the interviews were: to understand the professional perspective and vested interest of key stakeholders in DFM; to solicit views as to general effectiveness of DFM, especially to elicit constructive criticism; to invite suggestions for improving the DFM process.

16 individuals were interviewed: 8 DREs (covering: interior trim, exterior lighting, engines/gearbox), 3 manufacturing engineers, 2 industrial engineers, 1 dimensional engineer, 1 service engineer, 1 fastener 
engineer. Interviews typically lasted between 45 and 90 minutes. They were not tape recorded, instead detailed hand written notes were kept. Notes were reviewed each day and open-coded throughout the sampling process. The method of constant comparison was used to drive the development of theoretical categories. Data gathering in GT terminates when the flow of new ideas dries up (theoretical saturation) and the existing categories appear to provide a full account of "what is going on" in the field situation. The present researcher concluded his field work at the point when it was felt that the emergent theory was adequate to write a definitive report for the Company (including recommendations for change) and that further data gathering would produce only rapidly diminishing theoretical returns.

\section{FINDINGS AND GROUNDED THEORY}

\subsection{The Nature of Grounded Theory}

Before presenting the findings of the work, a few words will be said on the nature of Grounded Theory. Regarding "theory", the author is content to adopt the definition proposed by Corbin and Strauss who define theory as a coherent set of general concepts, systematically interlinked, that has explanatory power for some phenomenon of interest. The term grounded theory simply denotes theory built solely out of the data collected in a particular concrete setting (Glaser and Strauss, 1967). Academic theory relevant to that phenomenon is deliberately eschewed in the interests of stimulating creativity and nurturing unbiased analysis and interpretation. The corollary of this is that grounded theory applies only to the substantive setting studied and is a response to the intellectual and practical need that stimulated the enquiry. GT is not intended to generalise in the conventional sense, but this does not preclude generalisation. Grounded theory becomes progressively more general as more and more of the domain in question is explored (through theoretical sampling) until saturation point is reached. The more sites and settings that are examined, the broader will be the scope of the theory, but it remains substantive theory whilst it is limited to a particular empirical domain. Glaser and Strauss also discuss formal theory, by which they mean theory couched in such an abstract, conceptual way that it can be applied to any relevant substantive domain. In this work, given our specific focus on knowledge management, we are perforce limited to the production of substantive theory, however many sites and organisations we were to study.

The main features of the present theory are expounded in the rest of this section. Following some general comments on the workshops, the following two sub-sections list some of the main concepts that emanated from the two episodes of fieldwork. Given the focus on the efficacy of DFM as a KM process, these subsections simply enumerate the principal attitudinal concepts that emerged (via open coding) bearing on this question. From these concepts a set of categories reflecting generic success factors was derived. The final section presents the theory in terms of these categories, their inter-relationships and their bearing on the DFM process. A diagram will be used for this purpose. There is no dogma in GT as to how theories should be articulated; a diagram is used here for succinctness.

\subsection{Overall Comments on Workshop Effectiveness}

First we will begin with some general comments on the effectiveness of the DFM workshops. Considerable variation in the conduct of workshops was observed, and in their output in terms of the number and innovativeness of ideas and the degree to which these cohered into different design alternatives. In general, the workshops observed tended to produce proposals for marginal improvements (often addressing simple integration issues) rather than radical design ideas.

Measuring effectiveness in a formal way was inhibited as ideas and solutions were not consistently quantified in terms of financial and labour savings, nor were there any formal mechanisms in the divisions studied to track the progress of outcomes in terms of their implementation in the design process itself, and ultimately their impact at the manufacturing stage. Translating design ideas into practice was at the exclusive discretion of the DREs who were not held accountable for implementing the ideas; they could ignore the 
workshops with impunity. Examples were given where design proposals had been arbitrarily overruled by senior line managers. Although a report was produced for each workshop, these contained very little technical content. Recommendations regarding solutions were often unclear, and no information regarding final decisions and outcomes was communicated. Dissemination was limited to the workshop participants and the documents were not stored in an accessible repository.

Even though the sample was small, the motivating concerns of the research were borne out by these observations. The workshops did not appear to be effective as a source of design knowledge, the mechanisms for storing and disseminating this knowledge were crude, and there were no formal systems for monitoring the application of the knowledge.

\subsection{Factors Aadversely Influencing Workshop Effectiveness}

From workshop observations supported by informal interviews with participants, the following factors were identified as restricting the effectiveness of the workshops.

- Inadequate participation. The expectations of workshop participants as to the contribution that they were expected to make was commonly not explained to them in advance of workshops. Sometimes key interests were unrepresented, e.g. manufacturing engineers.

- Low commitment from DREs. Many DREs appear to be simply "checking the box", i.e. carrying out the workshop because the formal design process required them to, rather than because they had any real belief in the method. DREs appeared to be very protective of their designs and unwilling to expose them to criticism. They often failed to provide active leadership during the workshops; the DFM engineer tended to run the workshop in many cases.

- Poor preparatory work. DREs in particular were often ill-prepared for workshops (e.g. baseline designs were not available). In general, critical information (drawings, mock-ups) was not to hand, causing frustration and disillusionment. Very little use was made of past cases and previous lessons learned. Benchmarking was often not carried out, despite excellent vehicle "tear down" facilities.

- In-built conservatism. The need to re-use previous designs (i.e. "carry-over") limited the degree to which new design was possible; tight deadlines also restricted the freedom to explore new ideas. The risk of holding up the whole vehicle programme if an untried idea went wrong was a serious constraint. The tendency to see things in terms of prior knowledge and experience ("Cognitive set") was a major problem

- Dysfunctional workshop process: e.g. lack of clarity over evaluation criteria, inconsistent brainstorming approaches. Workshop goals were sometimes too abstract (seldom expressed quantitatively) and sometimes too complex (too many issues to be addressed). Short time formats (e.g. half day workshops) limited the information sharing phase of workshops and time for reflection and incubation. Vociferous elements often dominated workshops, unduly influencing the range and evaluation of solutions. Previous bad experiences of workshops was identified as a factor discouraging attendance and engendering unconstructive attitudes and poor preparation.

- Political behaviour. i.e. some delegates attended workshops to defend partisan interests or to advance tendentious causes, not to contribute positively.

\subsection{Formal Interviews}

The interviews conducted in the second episode of field work revealed two distinct perspectives: the DRE view, wherein feelings towards DFM were mixed, and the non-DRE perspective, wherein feelings were generally positive.

The DRE perspective: Of the 8 DREs interviewed, 3 were sceptical and 5 were supportive. In essence, the 3 sceptics saw DFM as a bureaucratic intrusion, that the issues it covered would be dealt with by any good engineer, as a natural part of the design process. These DREs typically felt that they had a good 
understanding of manufacturing issues and that face-to-face was the best way of progressing design work. At best DFM was a way of formally wrapping up a design, a useful "walk through" to get buy in for a design that was largely fait accompli. DFM was performed because it was mandatory (to get the tick in the box). All 3 expressed the view that DFM had probably once been useful when manufacturing awareness had been low amongst the engineering and design communities, but that it was largely redundant now. These DREs typically spent little time in pre-work, and expected little liaison with the DFM engineer following workshops.

The DREs who supported DFM did so because they appreciated the value of the multidisciplinary forum, i.e. of getting together a broad range of disciplines early in the design process to resolve a complex problem. DFM provided a useful way of addressing manufacturing, interfacing and packaging issues, and of obtaining pertinent specialist knowledge ("A DRE can't be expected to know everything"). Experiences of DFM in this group differed. Two DREs had had generally good recent experiences, in that workshops had generated interesting solutions that were being actively followed up. Another had found DFM to be valuable in the past, but in his current situation felt that it was used in a reactive way to solve problems once the design had been largely done. Whilst believing fervently in "up-front" engineering, he had recently used a workshop simply to get buy-in for a solution that he had already decided upon. The remaining two DFM proponents represented interesting cases. Both were involved in electrical systems and saw DFM as a way of finding out about the progress and trajectory of design solutions being made by other DREs, and hopefully influencing these decisions. In a sense, their perspective appeared much closer to that of the non-DRE group (see below).

The non-DRE view: Almost uniformly, the non-DRE stakeholders were very enthusiastic about DFM. In essence, they saw it as a forum which would allow them to have an influence on the design process, whether this be in relation to manufacturing concerns, new technology, and so on. A strong feeling came over from this group that many DREs were too protective of their designs and that they were a law unto themselves. DFM thus represented an opportunity for them "to have their voice heard". Whilst this group were very supportive of DFM, they nonetheless expressed some dissatisfaction, echoing many of the issues raised above. In addition, it was commented that workshops were often held too late in the design process, and that the workshop format was too rigid (e.g. too much time spent on brainstorming when this was not necessarily appropriate).

\subsection{The Grounded Theory}

Figure 1 depicts the final form of the grounded theory derived from the data and the concepts overviewed above. The semantics of the model have been kept deliberately simple. There are two principal ontological constructs: knowledge processes and perceived success factors, linked by arrows indicating dependencies. Two key KM processes are shown (double bordered boxes): knowledge generation and knowledge application. The distinction between knowledge creation and its application reflects a distinction consistently made, both in interviews and in the DFM documentation, between the creative process of generating design knowledge and the implementation activity entailed in carrying this through into the actual manufacturing process. Crudely, these categories (and their implicit subcategories) map well into models of the KM process as defined in the knowledge management literature (e.g. Bhatt, 2001; Beijerse 2000). The various success factors that were held to influence these two processes are also shown, in single-bordered boxes. Although these factors can clearly be divided into different sub-categories (e.g. cultural, organisational, technological), in the interests of simplicity a single notation is used given that they all belong to a common high level ontological class (i.e. success factor) and that their individual provenance can be readily inferred from the construct label. The diagram also indicates relationships (arrowed lines) between categories that were induced from the statements made by interviewees. At an abstract conceptual level, these links simply reflect the existence of a dependency of some sort amongst the success factors, and between the factors and the knowledge processes. For example, knowledge generation is indicated to depend on the commitment of DREs to the DFM process, and this commitment is in turn influenced critically by the degree of active support for DFM from their senior line managers. 


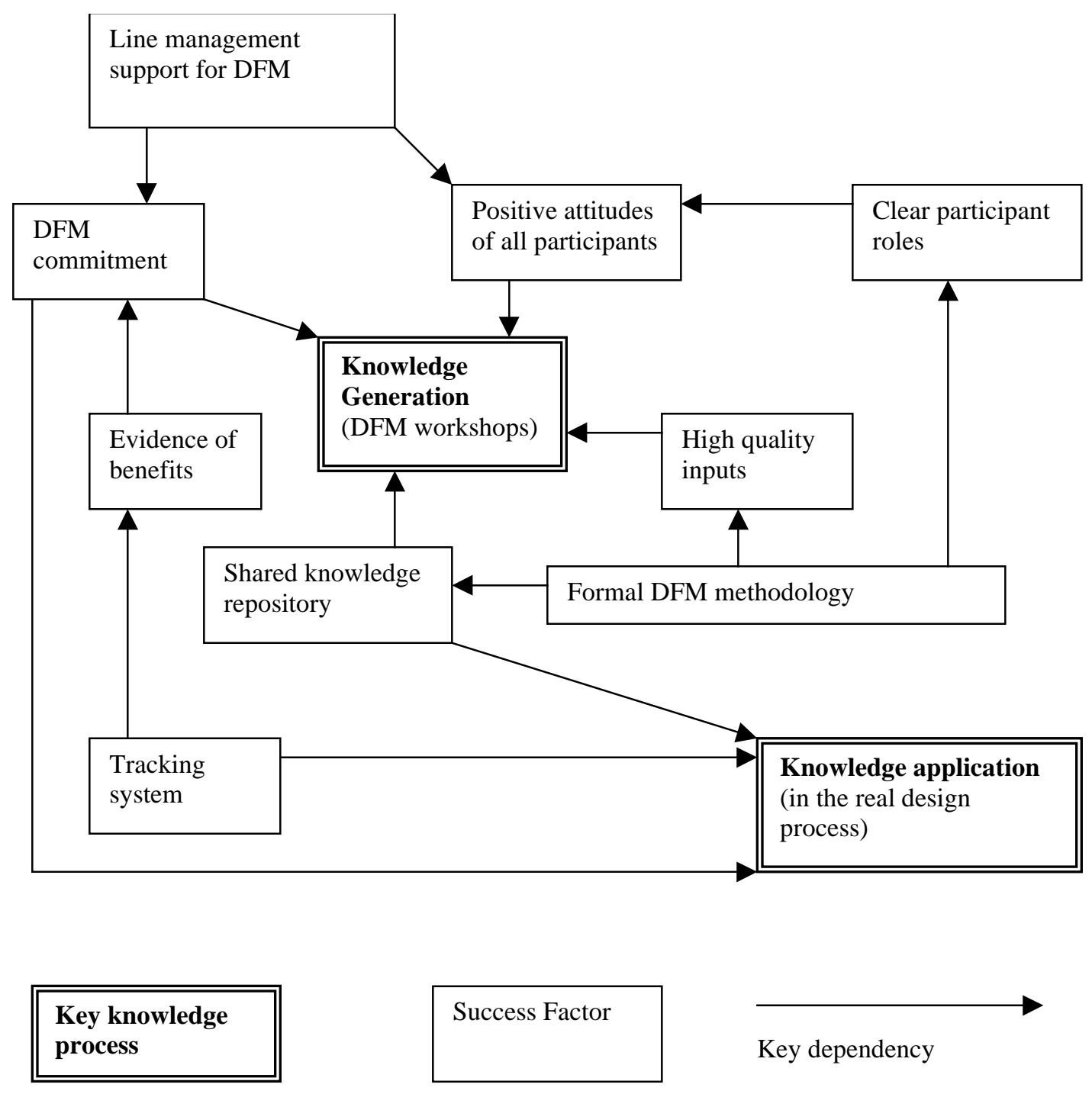

\section{Figure 1: Formal representation of the GT model of KM effectiveness}

The validity of the theory is attested in several ways. First, each of its main constructs can be tracked back to documentary evidence through the open-coding process (i.e. conceptual labelling of field notes, comprising paraphrased and quoted comments, scenario descriptions etc.). Second, the model has been formally presented to individuals in the company and has received their endorsement (e.g. the DFM user group). Thirdly, it has been informally triangulated in the course of individual interviews. Fourthly, the fact that it has provided a basis for practical recommendations, some of which have been implemented (see below), provides further endorsement.

\section{FROM GT TO ACTION}

It will be remembered that the purpose of the present research was not merely academic. Its practical aim was to generate recommendations for enhancing the DFM process to render it more effective as an instrument of knowledge management. In short, the project was an action research project. Action research can take on many guises (Baskerville and Wood-Harper, 1998); common to all these nuances is the idea of a 
shared endeavour involving academics and practitioners where the aim is to devise and evaluate an organisational intervention of some kind and to use this experience to build and/or test theory. The hallmark of AR is its highly collaborative ethos: i.e. the primary research questions are defined by practitioners and the role of the researcher is to facilitate the research work by assisting in methodological design, data collection, analysis and critical discussion. Action research therefore represents a true partnership of interests: on the Company side, assistance is provided by the researchers in addressing concerns of pressing importance to the business, for the academics it provides an opportunity to explore theoretically interesting problems in a real world setting. Grounded Theory fits into an action research scenario in a very natural way. It provides a systematic basis for developing and verifying theory (the research interest), and in designing and evaluating interventions (the practitioner interest). The degree to which the interventions are successful provides validation for the theory and may indicate areas where refinement is necessary. In this section, we will first review the recommendations for DFM improvements directly flowing from the GT, and go on to briefly summarise the actual process changes that have been put into effect

\subsection{Recommendations for Action}

A number of concrete recommendations to improve the effectiveness of DFM were proposed, covering the following areas:

- Process conformance. That a common DFM process be defined and that adherence to this should be formally monitored, especially prior to workshops to ensure that all participants were properly prepared and all key interests represented. It was specifically recommended that workshops should be cancelled if preparation or representation was deemed unsatisfactory and that DREs should be held accountable for this.

- Quality assurance. Improving documentation was argued to be critical, both before and after workshops. Standard templates should been defined and the quality of documents audited. Key inputs should be actively solicited before meetings. Regular surveys were proposed to evaluate the quality of DFM workshops. It was also recommended that feedback be solicited from participants at end of workshops regarding effectiveness, what went well, what didn't etc.

- Knowledge repository. It was strongly recommended that a central repository of case studies should be created allowing easy retrieval of relevant past design knowledge and the sharing of good practice. Evidence of benefits would be a critical part of this.

- Management issues. It was proposed that DREs and their line managers be made accountable for implementing DFM solutions and that incentive structures be changed to reward knowledge sharing. Central to this was the recommendation to define and implement a system of KM metrics. These would cover the whole DFM process, from the initial decision to hold the workshop, right through to the dissemination of design ideas and their implementation in practice. The metrics would address every critical step in the process, e.g. the quality of workshop input, manufacturing benefits (reduced part counts etc.)

\subsection{The Current Situation}

The author completed his fieldwork in the summer of 1999 and a report summarising his findings and recommendations was submitted to the DFM user group and the central support team. Since that time a number of process improvements have been implemented. The author would not wish to claim full credit for these changes. Many of the ideas had been under consideration for some time. Nonetheless, the author's work was highly influential in terms of reinforcing the need for these changes, in helping establish priorities and in refining and clarifying key issues.

The main interventions are as follows: a Global DFM process has been defined (for each process step, detailed "statements of work" and a set of standard document pro formas ("work tickets") have been 
produced); a tracking system used in one of the divisions (not studied in detail by the author) has now been rolled out across all divisions (this embodies a set of metrics enabling design ideas to be tracked from workshops right through to their implementation in the manufacturing process and their benefits assessed); a corporate DFM Web-site has also been created which will act as a central repository for all DFM workshop reports, including best practice exemplars and an effective search system in order to identify previous design knowledge relevant to a current design problem (cases will be described in narrative form, in order to capture informal as well as formal knowledge).

\section{DISCUSSION}

The aims of this paper were twofold. The first aim was substantive, to contribute to theory regarding knowledge management, in particular to shed light on the factors that can impede and undermine KM initiatives. The second aim was methodological, to demonstrate how grounded theory can enhance action research.

The grounded theory produced by this research provides a model of KM success, superficially akin to the factor models that have been produced elsewhere in the IS literature to highlight the key influences bearing on IS success. It differs, however, in the fundamental sense that it represents grounded theory unique to a single substantive context. It describes and explains the situation from which it was derived, and this situation alone. In other words, the model applies only to WWM; we can make no empirically-based claims for its generality beyond this. Further field work in other organisational sites (developing and extending the model through comparative analysis and theoretical sampling) is required to produce theory with more generalisable content. To a degree, this has happened already. Initially most of the data was gathered in only one division of WWM (Truck); as the work progressed, theoretical sampling in other divisions led to a richer set of categories that applied across the company as a whole. However, although saturation may have been reached in this particular organisation, further empirical work is undoubtedly necessary in other automobile companies (and beyond that in other manufacturing and non-manufacturing settings) before a complete theory for the substantive domain of KM is achieved.

Given that KM is a much researched area, the theory may nonetheless be usefully discussed in relation to this wider work, and in this sense its generalisability can be evaluated. Many aspects of the model resonate with the extant literature on KM. Three factors are highlighted as the key to KM success: repositories, commitment and formal process. These factors impinge on both knowledge generation and its application. The first factor stresses the need for a shared knowledge repository. Much of the KM literature focuses on technologies for knowledge management (Davenport et al., 1998). The absence of such an infrastructure seriously inhibited the efficacy of the DFM process in WWM. This underlines just how critical the role of technology is as an enabler of KM. The introduction of a shared DFM site can thus be expected to make a major impact.

But as many others have pointed out, technology is not a sufficient condition for effective KM whilst it is arguably a necessary one (Swan et al., 1999; Storey and Barnett, 2000). Changes in attitudes and working practices are critical to encourage knowledge sharing and the re-use of knowledge assets; Davenport (1998) refers to this as the need to establish a knowledge environment "conducive to more effective knowledge creation, transfer and use." This is an immeasurably more difficult problem than the infrastructural one. Storey and Barnet (2000) comment that the concerted determination to overturn deeply ingrained cultural practice is key, and irresolution in this regard lies behind many KM failures. Many others have made the same general point (e.g. Damodaran, 2000; McDermott and O'Dell, 2001). Cultural barriers in WWM are formidable. The design culture in the company is that of heroic individuals not team players; the traditions of WWM emphasise design ingenuity often at the expense of manufacturing efficiency. Whilst the measures by the company can be expected to make some inroads into this problem, primarily by demonstrating DFM efficacy and by making knowledge more available, organisational changes to attack the problem directly (e.g. through changing incentive and accountability structures to enforce the DFM approach) have been balked at so far. 
The third key element of the model is the need to strengthen the existing DFM methodology and encourage greater compliance. The author has argued elsewhere (Wastell and Kawalek, 2001) that methodology has considerable potential as a knowledge management device in multidisciplinary design teams. This is arguably more important than its traditional role as a control and co-ordination mechanism though it is seldom emphasised. By providing a shared linguistic and ontological framework, methodology affords a powerful structure for creating and binding together a community of practice (Lave and Wenger, 1991). Strengthening adoption and use of a formal DFM process has thus a key role to play in creating a vigorous DFM community within WWM. Moreover, the existence of a defined common process enables a system of metrics to be defined; without such a system, an organisation's knowledge processes are inherently unmanageable.

Turning finally to the methodological contribution of this work, the author has argued that GT has a valuable role to play in action research and indeed consulting practice. We have shown how grounded theory can strengthen mechanisms for building theory in AR and thereby add to its status and effectiveness as a research tool, taking some of the sting from the unfavourable criticisms that are often levelled at it. Potential is one thing, however, realisation another, and a change in attitude and self-understanding is also required amongst the AR community. In particular, a greater priority must be given to research over problem-solving. To this end, McKay and Marshall's (2000) thoughtful critique of AR is much to be welcomed, in particular their clarification that problem-solving methods (such as soft systems methodology) do not in themselves constitute AR. As well as strengthening the research side of AR, GT can also add rigour on the problemsolving side. Having a well-defined theoretical model is of considerable benefit in two main ways. First, it allows interventions to be designed in a systematic way to address points of maximum leverage. Second, it allows these interventions to be evaluated and enables outcomes to be understood. In WWM, a set of interventions based on the model was recommended and some of these have been put into effect. It is too early to evaluate the impact of these changes; they are the subject of on-going evaluation. Nonetheless the key point is that the model provides a framework which could be used to measure the impact of the changes and to understand their effectiveness (or lack of). On a pessimistic note, the model predicts that whilst some benefits will be yielded, the pivotal issue (of DRE commitment) is not yet being directly addressed and hence the gains might be expected to be marginal. The value of the model is that it indicates why this might be so and provides a framework to guide future improvement initiatives.

\section{ACKNOWLEDGEMENTS}

This work took place during the author's sabbatical at the Center for Innovation in Product Development at MIT. Financial assistance came from the NSF under Co-operative Agreement number EEC-9529140. The author gratefully acknowledges the support of David Bell for his role in initiating the work and his contribution to the research proposal. Warm thanks are also due to all who helped me at WWM, Steve Erskine and Michael Carter in particular.

\section{REFERENCES}

Baskerville, R. and Wood-Harper, A.T. (1998). Diversity of information systems action research methods. European Journal of Information Systems, 7, 90-107.

Bhatt, G.D. (2001). Knowledge management in organisations: examining the interaction between technologies, techniques and people. Journal of Knowledge Management, 5, 68-75.

Beijerse, R.P. (2000) Knowledge management in small and medium-sized companies. Journal of Knowledge Management, 4, 162-179.

Damodaran, L. and Olphert, Q. Barriers and facilitators to the use of knowledge management systems. Behaviour and Information Technology, 19, 405-413. 
Davenport, T. de Long, D.W. and Beers, M. (1998). Successful knowledge management projects. Sloan Management Review, 43-57.

Glaser, B.G. and Strauss, A.L. (1967). The discovery of grounded theory: strategies for qualitative research. De Gruyter, New York.

Goulding, C. (1998). Grounded theory: the missing methodology on the interpretivist agenda. Qualitative Market Research: an International Journal, 1, 50-57.

Gupta, A.K. and Govindajaran, V. (2000). Knowledge management's social dimension: lessons from Nucor Steel. Sloan Management Review, February, 71-80.

Holsapple, C.W. and Joshi, K.D. (2000). An investigation of factors that influence the management of knowledge in organisations. Journal of Strategic Information Systems, 9, 235-261.

Lave, J. and Wenger, E. (1991) Situated learning and legitimate peripheral participation, Cambridge University Press, Cambridge.

Lynn, G.S., Reilly, R. and Akgun, A.E. (2000) Knowledge management in new product teams: practices and outcomes. IEEE transactions on Engineering Management, 47, 221-231.

McDermott, R. and O'Dell, C. (2001). Overcoming cultural barriers to sharing knowledge. Journal of Knowledge Management, 5, 76-85.

McKay, J. and Marshall, P. (2001). The dual imperatives of action research. Information Technology and People, 14, 46-59.

Ramesh, B. and Timawa, A. (1999). Supporting collaborative process knowledge management in new product development teams. Decision Support Systems, 27, 213-235.

Schultze, U. and Boland, R. (2000). Knowledge management technology and the reproduction of work practices. Journal of Strategic Information Systems, 9, 193-212.

Shani, A.B., Sena, J.A. and Stebbins, M.W. (2000). Knowledge work teams and groupware technology: learning from Seagate's experience. Journal of Knowledge Management, 4, 111-124.

Storey, J. and Barnett, E. (2000). Knowledge management initiatives: learning from failure. Journal of Knowledge Management, 4, 145-156.

Strauss, A. and Corbin, J. (1998). Basics of qualitative research. Sage, London.

Swan, J., Newell, S. Scarborough, H. and Hislop, D. (1999). Knowledge management and innovation: networks and networking. Journal of Knowledge Management, 3, 262-275.

Wastell, D. and Kawalek, P. (2001). Designing alignment and improvising change: experiences in the public sector with the SPRINT methodology. Proceedings of ECIS 2001, Bled. 